\title{
Yeast metabolic state identification using micro-fiber optics spectroscopy
}

\author{
Silva, J.S. ${ }^{1}$, Castro, C.C. ${ }^{1}$, Vicente, A.A. ${ }^{1}$, Tafulo, P $^{2}$, \\ Jorge, P. A. S $^{2}$ and Martins, R.C. ${ }^{3 *}$ \\ ${ }^{1}$ Centre of Biological Engineering, Minho University, Campus of Gualtar, 4710-057 Braga-Portugal \\ ${ }^{2}$ INESC Porto, Rua do Campo Alegre 687, 4169007 Porto, Portugal \\ ${ }^{3}$ Molecular and Environmental Biology Centre, \\ Minho University, Campus of Gualtar, 4710-057 Braga-Portugal
}

\begin{abstract}
Saccharomyces cerevisiae morphology is known to be dependent on the cell physiological state and environmental conditions. On their environment, wild yeasts tend to form complex colonies architectures, such as stress response and pseudohyphal filaments morphologies, far away from the ones found inside bioreactors, where the regular cell cycle is observed under controlled conditions (e.g. budding and flocculating colonies). In this work we explore the feasibility of using micro-fiber optics spectroscopy to classify Saccharomyces cerevisiae S288C colony structures in YPD media, under different growth conditions, such as: i) no alcohol; ii) $1 \%(v / v)$ Ethanol; iii) $1 \%(v / v) 1$-butanol; iv) 1 $\%(v / v)$ Isopropanol; v) $1 \%(v / v)$ Tert-Amyl alcohol (2 Methyl-2-butanol); vi) 0,2 \% (v/v) 2-Furaldehyde; vii) $5 \%(w / v)$ 5 (Hydroxymethyl)-furfural; and viii) $1 \%$ (w/v) (-)-Adenosine3', 5'cyclic monophosphate. The microscopy system includes a hyperspectral camera apparatus and a micro fiber (sustained by micro manipulator) optics system for spectroscopy. Results show that micro fiber optics system spectroscopy has the potential for yeasts metabolic state identification once the spectral signatures of colonies differs from each others. This technique associated with others physico-chemical information can benefit the creation of an information system capable of providing extremely detailed information about yeast metabolic state that will aid both scientists and engineers to study and develop new biotechnological products.
\end{abstract}

Keywords: Yeast metabolic state, Spectroscopy, Micro fiber optics.

\section{INTRODUCTION}

Studies show that $S$. cerevisiae can form complex colony structures with an apparent cell specialization. Colonies of wild yeasts can contain all the varieties of cells, from witch the mostly known are the diploid, haploid, hyphae form (diploid or haploid) and ascus (spore); opposing to the most well known yeast cell cycle - the budding yeast. Furthermore, it is known that $S$. cerevisiae can undergo changes in their replicative patterns and morphologies, according to environmental conditions (i.e., deleterious), to produce elongated cells joined-together in filaments (1) and colonies can signal each other (2). The yeast-form and filamentous-form cell cycles are similar but, according to (3), in yeast-form growth, daughter cells are smaller than its mother and must undergo a period of further growth (in phase G1) before starting a new cell cycle ('asymmetric cell division'). On the other hand, filament-form cells have a 'symmetric cell division', once after mitosis and cell division, both mother and daughter cells are equal-sized and bud emerge starts in both cells. Furthermore mitochondrial mass and chitin deposition increases in filaments. The filaments walls have greater strength and rigidity than those of yeast-form cells, which has been suggested as a mean of penetration of solid media because yeast lack natural mobility. The transcription of all of genes also decreases in filament forming yeast, and therefore has been proven difficult to find a direct transcriptomic relationship (1).

Filament formation can be induced by nitrogen starvation or limitation (4), growth on a poor nitrogen source (5) or growth in the presence of low concentrations of fusel alcohols (end-products of cells catabolism). In the case of nitrogen starvation or limitation, filamentation can be explained as a foraging response because yeast is non-motile and cannot move to search for a richer supply of nutrients, it can only grow to explore its surroundings (6). Filamentation has

International Conference on Applications of Optics and Photonics, edited by Manuel F. M. Costa,

Proc. of SPIE Vol. 8001, 80013H · C 2011 SPIE · CCC code: 0277-786X/11/\$18 · doi: 10.1117/12.892262

Proc. of SPIE Vol. $800180013 \mathrm{H}-1$ 
also been induced by superior alcohols and AMPc, and has been argued that these may act as communication molecules between different yeast, allowing for the colony to synchronize its development, a phenomena known as 'quorumsensing'. Therefore, the yeast colony state and its dynamics are not yet explained. In order to understand this phenomena, an non-targeted, holistic and high-output approach is needed in order to gather the maximum information as possible to understand colony dynamics and yeast communication. In this sense, the use of spectroscopy in conjunction with other techniques may allow to implement real-time and non-destructive methodologies that can explain the transcriptomics and metabolomics processes happening in yeast cellular communities.

Microorganisms traditional identification methods are supported by morphological and growth capacity in selective media (7). The use of high-output methodologies to increase analysis capacity, such as mass spectroscopy, PCR and spectroscopy are becoming popular, not only because of the time needed for an effective identification, but more importantly because these methods are multivariate, which allows to obtain vast amounts of information in one measurement (8). Spectroscopy is a simple, precise, rapid, multivariate and non-destructive technique. Spectra is proportional to the chemical composition of the analite, acting as a non-destructive methodology capable of both fingerprint and quantifications. In this case, spectroscopy is may be able to classify the invariable structure of yeast, the metabolism and cell communication. Cells morphology is a visible expression of microorganisms physiology and metabolism (9). Different morphologies characterize different proteomic composition and perhaps different metabolism states that can be differentiated by UV-VIS-SWNIR spectroscopy.

Many spectroscopy techniques have been used for microorganisms identification, where NIR and Raman spectroscopy are the most popular $(10,11,12)$. Recently, a previous study revealed that UV-VIS-SWNIR is also a highly accurate spectroscopy method for microorganisms identification (13). UV spectroscopy records electronic transitions between electron energy levels from molecular levels in the UV-VIS region depend upon the energy involved. For any molecular bound (sharing a pair of electrons), orbitals are a mixture of two contributing orbitals $\sigma$ and $\pi$, with corresponding anti-bounding orbitals $\sigma^{*}$ and $\pi^{*}$, respectively. Some chemical bounds present characteristic orbital conditions, ordered by higher to lower order energy transitions: i) alkanes $\left(\sigma \rightarrow \sigma^{*} \$ ; 150 \mathrm{~nm}\right)$; ii) carbonyls $\left(\sigma \rightarrow \pi^{*}\right.$; $170 \mathrm{~nm})$; iii) unsaturated compounds $\left(\pi \rightarrow \pi^{*} ; 180 \mathrm{~nm}\right)$; iv) molecular bounds to $\mathrm{O}, \mathrm{N}, \mathrm{S}$ and halogens $\left(n \rightarrow \sigma^{*} ; 190 \mathrm{~nm}\right)$; and v) carbonyls $\left(n \rightarrow \pi^{*} ; 300 \mathrm{~nm}\right)$. As most UV-VIS spectrometers yield a minimum wavelength of $200 \mathrm{~nm}$, this technique has been considered to provide lower information in terms of functional groups when compared to IR, because spectral differences mostly attributed to conjugated $\pi \rightarrow \pi^{*}$ and $n \rightarrow \pi^{*}$ transitions $\backslash$ cite $\{14,15,16)$.

Many organic molecules present conjugated unsaturated and carbonyls bounds, such as aminoacids, phospholipids, free fatty acids, phenols and flavonoids, peroxides, peptides and proteins, sugars and their polymers absorve in these bands. UV-VIS not only records the effect of electron excitation, but also the effect of return to lower orbitals, which result in vibrational and rotational modes, increasing the characteristic spectra of biological materials. This effect enhances photochemical reactions and fluorescence which are important features for microbiological identification $(14,17,18)$ and may help to identify metabolic states of yeast. Many biological molecules also present chromophore groups, which increase the absorption in the UV-VIS region, such as: nitro, nitroso, azo, azo-amino, azoxy, carbonyl and thiocarbonyl $(17,18)$. Moreover the sensitivity of today's spectrometers has highly increased, being possible to obtain low noise to signal ratios which expands the detection limits. LWUV-VIS-SWNIR has as main advantage, the minimization of liquid water absorbance and effect of temperature. Furthermore as state of the art spectrometers also include high frequency vibrational infrared (SWNIR), it is also possible to obtain important information on water, fats and proteins $(19,20)$.

The main objective of this exploratory work is to determine if micro-fiber optics spectroscopy is a suitable methodology that may be used to recognize the state of Saccharomyces cerevisiae S288C colony structures in YPD media by spectral signal processing to obtain discrimination among different induced metabolism, cellular communication and morphology. 


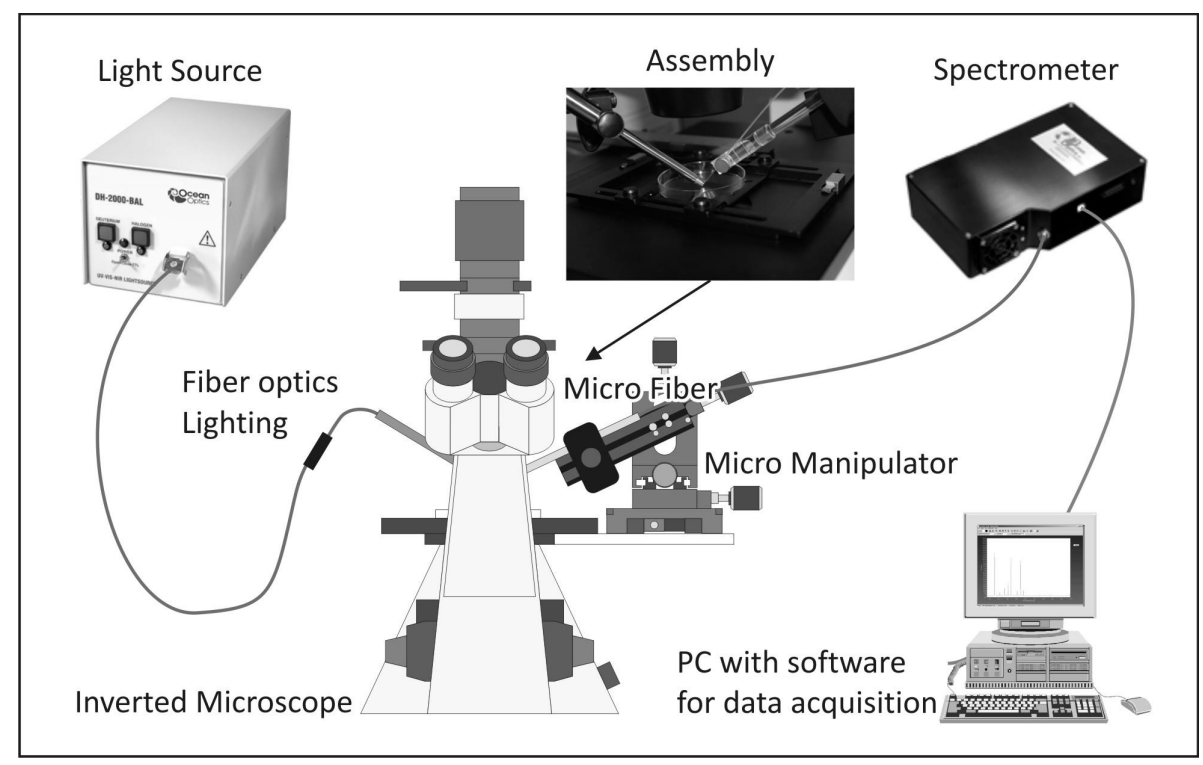

Figure 1. Scheme of the assembly of the spectroscopy equipment

\section{MATERIALS AND METHODS}

\subsection{Sample preparation}

Saccharomyces cerevisiae S288C was obtained from the microbiological collection of the Molecular and Environmental Biology Centre at the University of Minho. The incubation was performed in YPD broth medium (Sigma Aldrich - ref. Y1375) during 12 hours at $30^{\circ} \mathrm{C}$ under constant agitation $(150 \mathrm{rpm})$. S.cerevisiae S288C was inoculated on erlenmeyer flask $(100 \mathrm{ml})$ with $50 \mathrm{ml}$ of YPD growth medium, fitted with glycerol-locks, using different growth conditions. The inoculum was composed of S. cerevisiae S288C with a concentration of 16x106 cells / ml of YPD growth media and was incubated at $30^{\circ} \mathrm{C}$ during $24 \mathrm{~h}$. Studied conditions were: without alcohol, ethanol (Riedelde Han ref. 32221), 1- butanol (Sigma Aldrich - ref. BT105), isopropanol (Sigma Aldrich - ref. 190764), tert-Amyl alcohol, (2methyl-2-butanol) (Sigma Aldrich - ref. 152463), 2-furaldehyde (Sigma Aldrich - ref. 18591), 5 (hydroxymethyl)furfural (HMF) (Sigma Aldrich ref. 110889) and (-)-adenosine3', 5'cyclic monophosphate (AMPc) (Sigma Aldrich ref. A 0670) (21). Growth medium present the following constitutions: YPD broth medium (Sigma-Aldrich ref. Y1357): 10 g.1-1 Yeast extract , 20 g.l-1 Peptone and 20 g.l-1 Glucose (21). For each growth condition were inoculated six different erlenmeyer flasks, which were analyzed at different times after inoculation: T0 -0 hours; T1 -4 hours; T2 - 6 hours; T3 -10 hours; T4 -14 hours and T5 -24 hours.

\subsection{Spectroscopy}

Figure 1 presents a schematic of the experimental apparatus. Spectroscopy measurements were performed using the fiber optic spectrometer QE6500 (1044 x 64 pixels, 200-1100 nm) connected to a micro fiber optic (22). The micro fiber is attached to an inverted microscope using a micro manipulator. In parallel with the micro fiber is a transmission probe TP-300-UV-VIS connected to a light source DH-2000 which transmits light in the sample that will be captured by the micro fiber (22). Measurements were performed at room temperature $(18 \pm 2 \mathrm{oC})$, and the deuterium and halogen light sources were let to stabilize for 20 and $15 \mathrm{~min}$, respectively. The dark spectrum was recorded and measurements were taken with linear and electric dark correction. Measurements of light are plotted into a multivariate control chart to evaluate reproducibility of the light source. Twenty spectrum replicates were recorded under each of the light sources for all the samples. Furthermore, spectra were obtained inside a dark chamber box designed to isolate the environmental light. 


\subsection{Micro fiber}

The micro probes were prepared using silica optical fibers with 550/600 $\mu \mathrm{m}$ core/cladding diameters and a $1 \mathrm{~mm}$ plastic buffer acquired from Thor labs (0.22 NA UV-Vis). Segments of fiber with $16 \mathrm{~cm}$ were cut and thoroughly polished in both ends. In one of the tips, $2 \mathrm{~cm}$ of the protective buffer was striped out and the fiber was cleaned with acetone.

To obtain the micron sized tips an etching solution was prepared. A solution of $48 \%$ HF was poured into a vial followed by a small amount of silica oil. Due to the difference in densities the two reagents don't mix creating a boundary between them. The striped fiber was then inserted into the etching solution through the silica oil layer and into the HF. At the oil/acid interface a meniscus was formed with a fixed angle and a certain high that depends on the fiber diameter. The small fiber segment immersed into the acid was gradually etched decreasing its diameter and the meniscus height. After $\sim 5 \mathrm{~h}$ the fiber immersed in HF was totally consumed and a sharp tip was formed by the interface meniscus (tip diameter $\sim 10 \mu \mathrm{m})$. The fiber probes where then inserted into the micro manipulators and connected to the Spectrometer using a temporary SMA connector from Ocean Optics and used to collect the spectra from specific locations of the sample.

\subsection{Spectral Analysis}

\section{Robust mean scattering correction}

The collected spectra were smoothed by using a Savisky-Golay filter (length $=4$, Order $=2$ ) (23) and subsequently, pre-processed using a modified robust multiplicative scatter correction algorithm (RMSC) $(24,25,26)$ : $x \operatorname{corr}=x b+a=x$ ref. The $a$ and $b$ coefficients are computed iteratively by minimizing the error: $e j=b x j+a-x$ ref; where $\mathrm{xj}$ is the $\mathrm{j}$ sample spectrum and $\mathrm{xref}$ is a reference spectrum. The RMSC algorithm is based on the application of the robust least squares method to determine the $a$ and $b$, ensuring that spectral areas that do not correspond to scattering artifacts are not taken into consideration. The robust least squares algorithm is implemented by the re-weighted least squares with the weights computed using the Huber function. The algorithm high breakdown point (50\%) ensures that existent outliers will not distort the model fitting and thus, scatter correction parameters are determined only with the consistent spectral areas. The iterative algorithm can be described, briefly as follow: 1) set the reference spectrum (xref) equal to the sample spectrum closest to the median spectrum; 2) correct the remaining sample spectrum by applying the above described robust least squares procedure; and 3) recompute the median spectrum and iterate until convergence.

\section{Singular value decomposition}

Singular value decomposition (SVD) is a blind signal decomposition technique widely used in spectroscopy data, where the corrected spectrum (Xcorr) is decomposed in order of magnitude of the variation directions in the variable space (wavelengths) when centered or scaled, they decomposition is called the Principal Component Analysis. Generally, most variability is captured in the first principal components (PC), where as, in good signal to noise spectral data, noise is captured in the last decompositions. Therefore a spectrum can be decomposed as: $x$ cor $=x+\varepsilon x$; where $x$ is the signal and $\varepsilon \mathrm{X}$ is the estimated noise of $\mathrm{X}$. This decomposition is possible to be performed by singular value decomposition (SVD):

$$
\mathrm{x}=\mathrm{USV}^{\top}
$$

where US are the scores, VT the loadings and the S singular values, respectively $(27,28,29)$.

To distinguish between the number of relevant decompositions, a randomization test is performed on the original matrix $(\mathrm{x})$ to determine the number of relevant singular values (30). In this research, 500 randomizations were performed by rotating the spectral intensity value at the same wavelengths among the different samples, to avoid violating the spectral continuity. By comparing the singular values of randomized spectrum with the original spectrum, the number of independent singular values and decompositions that discriminate the different microorganisms spectrum are obtained, so that:

$$
\mathrm{x}=\mathrm{US}_{\mathrm{rel}} \mathrm{V}_{\mathrm{rel}} \mathrm{T}
$$

where $\mathrm{US}_{\text {rel }}$ and $\mathrm{V}_{\text {rel }} \mathrm{T}$ are the statistically relevant scores and loading of $\mathrm{x}$, respectively. 
(a)

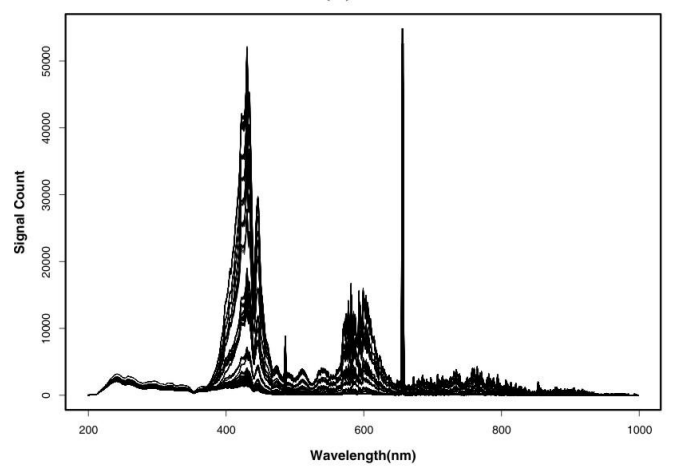

(c)

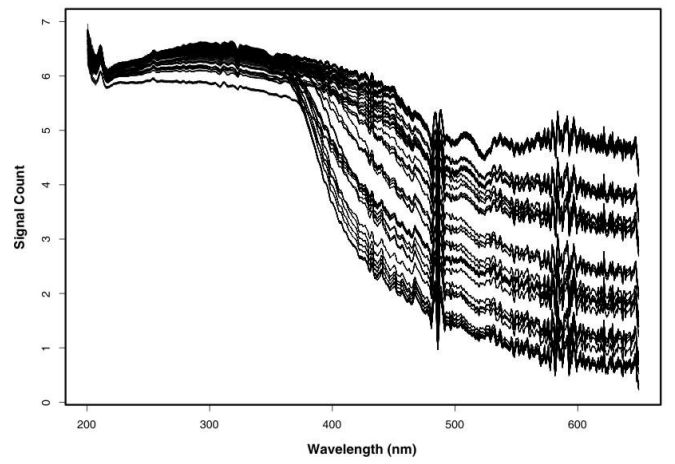

(b)

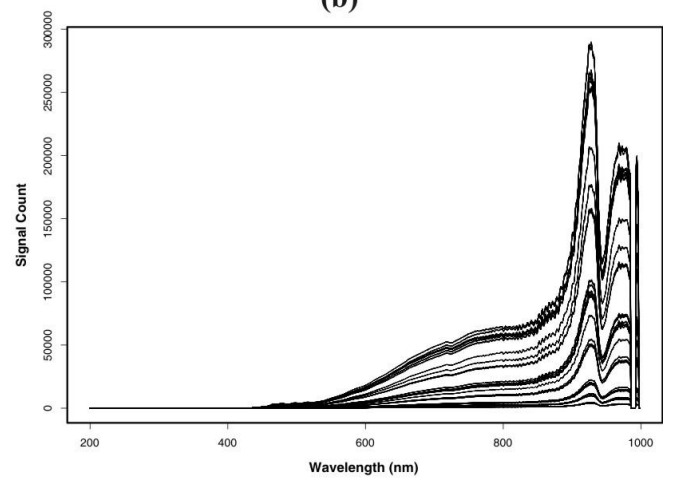

(d)

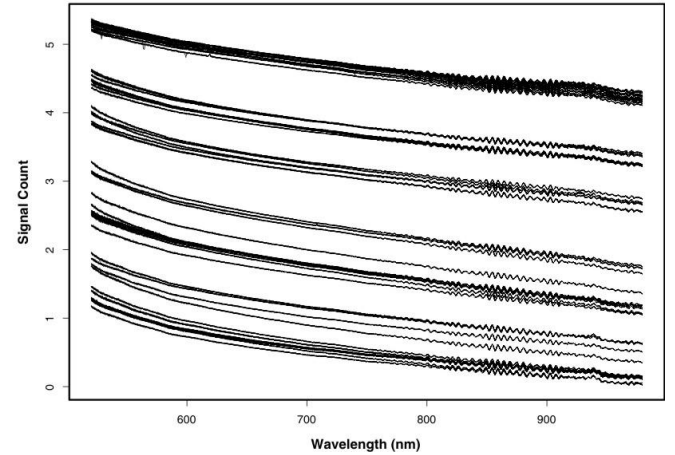

Figure 2. Samples Spectra: (a) raw spectra LWUV-VIS; (b) raw spectra VIS-SWNIR;

(c) absorbance spectra LWUV-VIS and (d) absorbance spectra VIS-SWNIR.

\section{RESULTS AND DISCUSSION}

\section{Spectral Absorbance}

Figure 2 presents LWUV-VIS and VIS-SWNIR yeast spectra for different growth medium conditions. In Figure 2 (a) and (b) we can observe that there is a wide variation of signal intensity throughout the growth of yeast. It is possible to assess in the absorbance spectra (Figure 2 (c) and (d)) that samples are directly distinguishable by the intensity and spectral shape. In this signal samples can be distinguishable in the wavelength interval of 200-650 $\mathrm{nm}$ and 550-990 $\mathrm{nm}$ in the LWUV-VIS and VIS-SWNIR, respectively. It is possible to observe that spectra signatures for each growth medium conditions are different and well distinguish, and therefore it is reasonable to assume that most of the information obtained in the spectra is coherent with the yeast metabolomic state.

\section{Singular value decomposition analysis}

Figure 3 (a) present relevant scores plot in the 2 PC's for LWUV-VIS absorbance, totalizing $98 \%$ of spectral variance with discriminate power (PC1 (92,3\%), PC2 (5,7\%)). Figure 3 (b) show the relevant scores for VIS-SWNIR, totalizing 99,93\% of spectral variance (PC1 $(99,90 \%)$ and PC2 (0,03\%). Both LWUV-VIS and VIS-SWNIR plots evidence optical properties of the different growth conditions, being this mostly described by the 1st principal component. The second principal component mainly distinguish sampling time of each growth condition. However, for the LWUV-VIS it distinguishes the growth condition on the action of the HMF and Isopropanol.

The light source LWUV-VIS provides a better differentiation of growth conditions. It is possible to observe that the samples of growth over time are more grouped in relation to growth condition. 
(a)

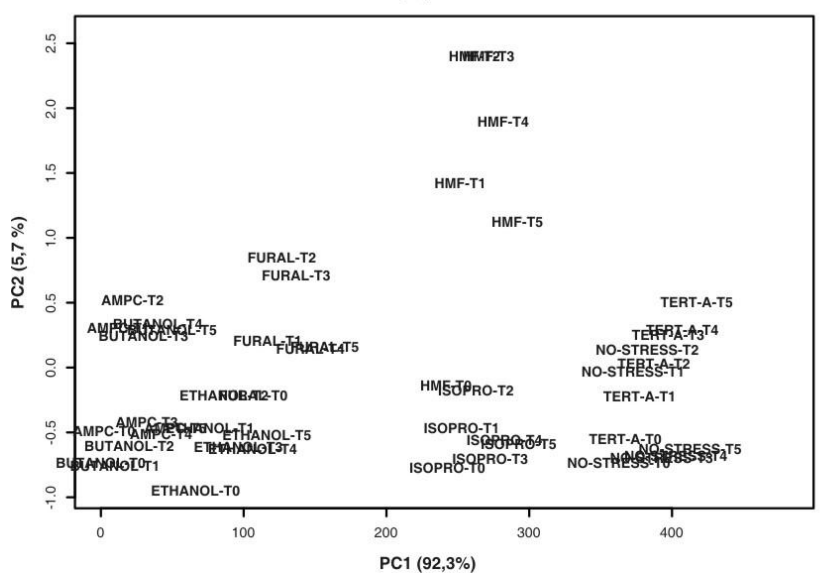

(b)

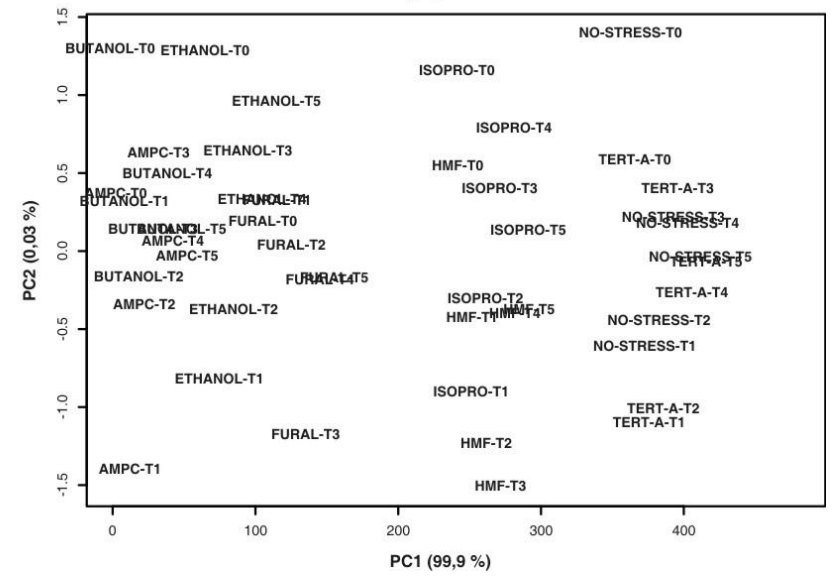

Figure 3. Absorbance spectra PCA analysis: (a) LWUV-VIS Gabriel Plot (PC1 (92,30\%), PC2 (5,70\%); and (b) VIS-SWNIR Gabriel Plot (PC1 (99,90\%), PC2 (0,03\%);

(a)

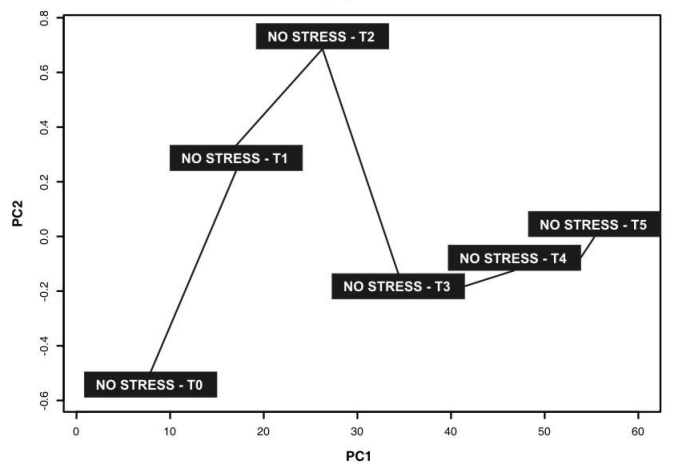

(c)

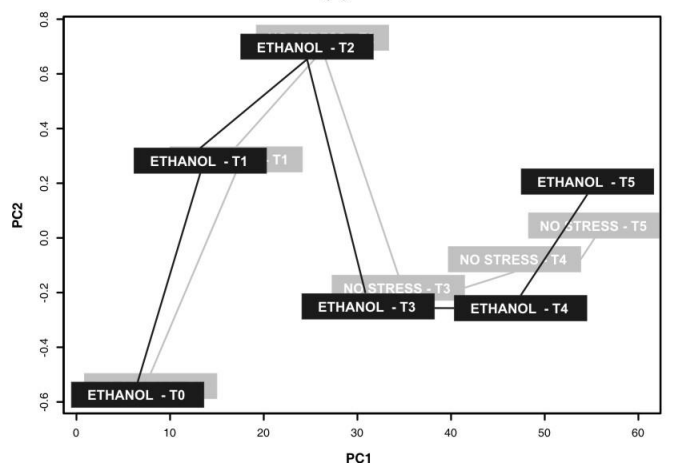

(b)

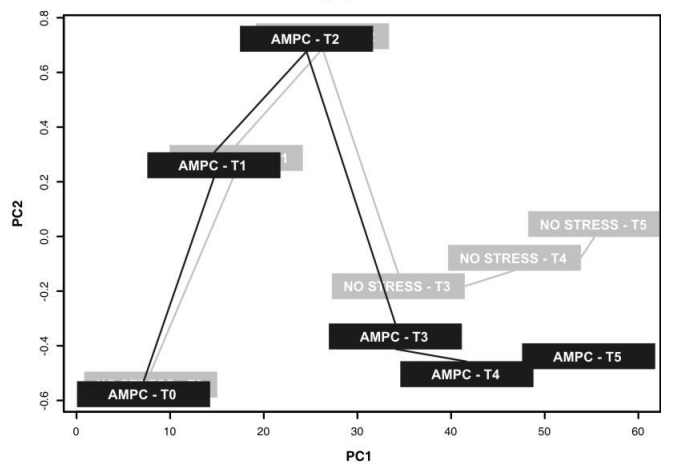

(d)

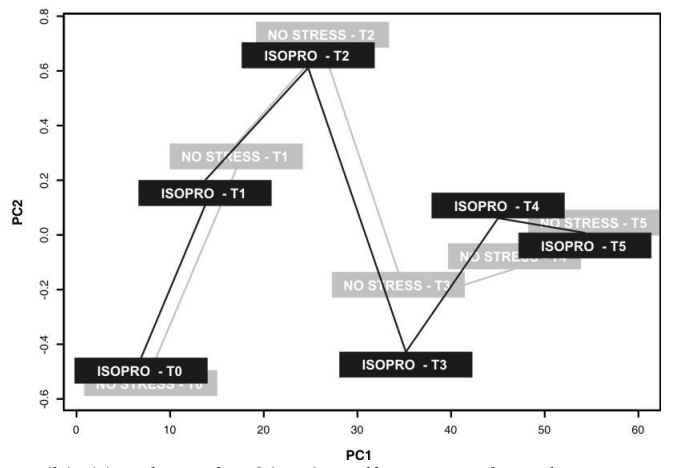

Figure 4. Similar spectra growth profiles: (a) no stress; (b) (-)-adenosine3', 5'cyclic monophosphate; (c) ethanol and (d) isopropanol

Figure 4 (b), (c) and (d) shows the spectral profile of each condition of growth over time that are similar to the normal growth of yeast (figure 4 (a)). The growth condition AMPc is the most similar to the normal, but only with chemical data of the samples is that you can justify these facts. Figure 5 shows the conditions that have a very different spectra growth profiles. It is likely that these samples have a large metabolic state variation, but only with chemical data will be possible to confirm. It should be noted that the 5 (Hydroxymethyl)-furfural (HMF) and the 2-Furaldehyde (Fural) have very similar profiles. 
(a)

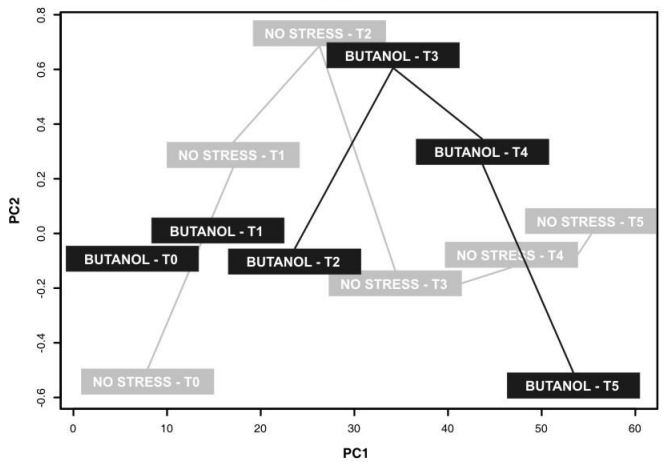

(c)

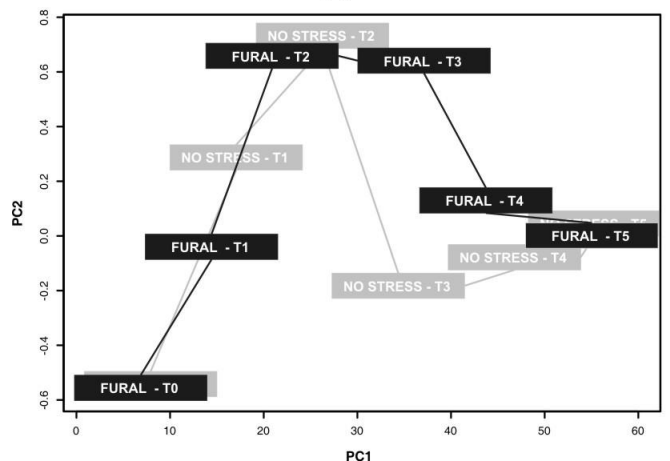

(b)

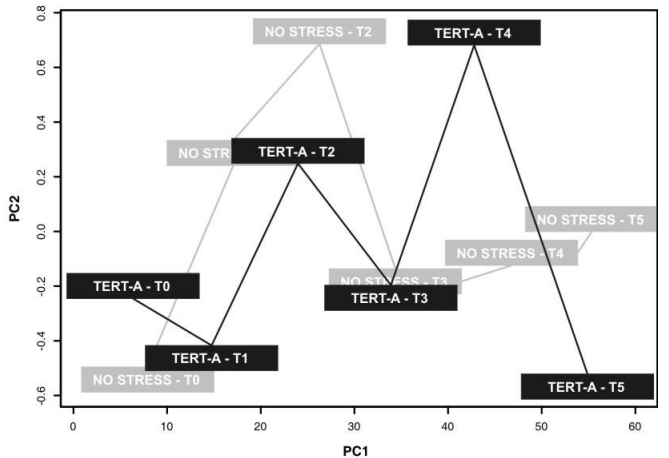

(d)

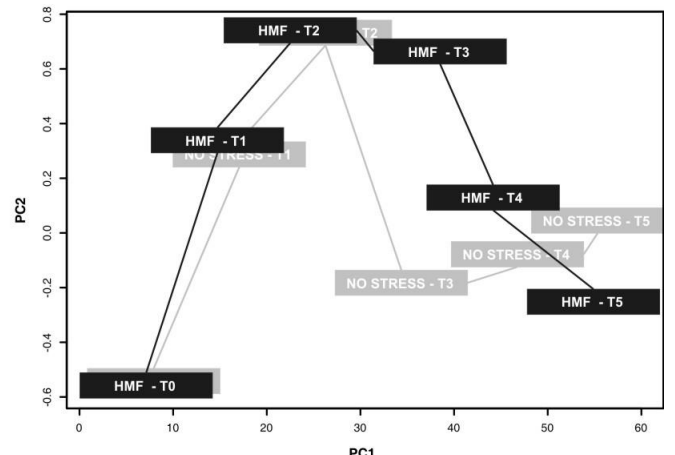

Figure 5. Different spectra growth profiles: (a) butanol; (b) tert-amyl alcohol;

(c) 2-furaldehyde and (d) 5 (hydroxymethyl)-furfural

\section{CONCLUSIONS}

This work has shown that after appropriate preprocessing and signal classification, micro fiber optics system spectroscopy is a high resolution technique capable of attaining extremely interesting possibilities in nondestructive metabolomics in the near future. Further insights will be gained when spectral information is deeper understood, not only by correlating with other high resolution analytical chemistry and molecular biology techniques.

\section{ACKNOWLEDGEMENTS}

The author J.S. Silva tanks to the Fundação para a Ciência e Tecnologia for the $\mathrm{PhD}$ grant SFRH/BD/48616/2008. Paula Tafulo acknowledges the support of FCT, fellowship SFRH / BD / 68924 / 2010. Part of this research was funded by the project PTDC/BIO/69310/2006.

\section{REFERENCES}

1. Dickinson, J., "Filament formation in Saccharomyces cerevisiae - a review", Folia Microbiol., 53(1), 3-14, (2008).

2. Palkova, Z. and Vachova, L., "Ammonia signaling in yeast colony formation".

3. Kron, S. and Gow, N.. "Budding yeast morphogenesis: gignaling, cytoskeleton and cell cycle", Curr.Opin.Cell Biol., 7, 845$855,(1995)$.

4. Rua, D., Tobe, B., and Kron, S., "Cell cycle control of yeast filamentous growth”, Curr.Opin.Microbiol., 4, 720-727, (2001).

5. Dickinson, J., "Irreversible formation of pseudohyphae by haploid Saccharomyces cerevisiae", FEMSMicrobiol. Lett., 119, 99-104, (1994). 
6. Gimeno, C. J., Ljungdahl, P., Styles, C., and Fink, G., "Unipolar cell divisions in yeast Saccharomyces cerevisiae lead to filamentous growth: regulation by starvation and ras", Cell, 68, 1077-1090, (1992).

7. Gerard, J., Berdell, R., and Christine, L., [Microbiology: An Introduction], Benjamin Cummingsc, New York, (2006).

8. Rosah, P., Hard, M., Schmitt, M., Peschke, K. D., Ronneberger, O., Burkhart, H., Mutzkus, H. W., Laukers, M., Hofer, S., Thiele, H., and Popp, J., "Chemotaxonomic identification of simple bacteria by micro-raman spectroscopy: application to clean-room-relevant biological contamination", A\&EnvMicro, 71(3), 1626-1637, (2005).

9. Treskatis, S., Orgeldinger, V., Wolf, H., and Gilles, E. D., "Morphological characterization of filamentous microorganisms in submerged cultures by on-linedigital image analysis and pattern recognition”, Biotechnol Bioeng., 53(2), 191-201, (1997).

10. Stuart, B., [Infrared Spectroscopy: Fundamentals and Applications], John Wiley \& Sons, Ltd, London, (2004).

11.Dziuba, B., Babuchowski, A., Naleczb, D., and Niklewicz, M., "Identification of lactic acid bacteria using ftir spectroscopy and cluster analysis", International Dairy Journal, 17, 183-189, (2007).

12. Bhatta, H., Goldys, E., and Learmonth, R., "Rapid identification of microorganisms by intrinsicfluorescence. In Imaging, Manipulation, and Analysis of Biomolecules and Cells", Fundamentals and Applications III, SPIE, (2005).

13. Silva, J., Martins, R. C., Vicente, A., and Teixeira, J., "Feasability of yeast and bacteria identification using UV-VIS-SWNIR difusive reflectance spectroscopy", BIOSIGNALS 2008: Proceedings of the First International Conference on Biomedical Electronics and Devices, 1, 25-32, (2008)

14.Levine, I., [Molecular Spectroscopy], John Wiley \& Sons, New York, (1975).

15.Denney, R. and Sinclair, R., [Visible and ultraviolet spectroscopy], John Wiley \& Sons, London, (1987).

16.Perkauparus, H., Grinter, H., and Therfall, T., [Uv-Vis spectroscopy and its applications], Springer-Verlag, New York, (1994).

17. Coyle, J., [Introduction to Organic PhotoChemistry], John Wiley \& Sons, London, (1989).

18. Klessinger, M. and Michl, J., [Exited states and photochemistry of organic molecules], VCH Publishers, New York, (1995).

19. Burns, D. and Ciurczak, E., [Handbook of near infrared analysisI] Marcel Dekker, Inc, New York, (2001).

20.Devices, A. S., Near-ir absorption bands, (2005).

21. Sigma-Aldrich Quimica, S., "Sigma, Life Science: Produtos para Investigação em Ciências da Vida, 2008-2009”, SigmaAldrich, Portugal, (2008).

22. Ocean Optics, [Catalog of products: Spectrometer, Accessories and More], Ocean Optics, The Netherlands, (2011).

23. Savizky, A. and Golay,M., "Smoothing and differentiation of data by simplified least squares procedures", Analytical Chemistry, 36, 1627-1639, (1964).

24.Gallager, N. B., Blake, T., and Gassman, P., “Application of extended inverse scattering correction to mid-infrared reflectance of soil", Journal of Chemometrics, 19, 271-281, (2005).

25. Martens, H. and Stark, E., "Extended multiplicative signal correction and spectral interference subtraction: new preprocessing methods for near infrared spectroscopy”, Journal of Pharmaceutical and Biomedical Analysis 9, 625-635, (1991)

26. Martens, H., Nielsen, J. P., and Engelsen, S. B., "Light scattering and light absorbance separated by extended multiplicative signal correction. application to near-infrared transmission analysis of powder mixtures", Analytical Chemistry 75(9), 394404, (2003).

27. Jolliffe, I., [Principal Component Analysis], Springer, New York, (1986).

28. Krzanowski, W. J., [Principles of Multivariate Data Analysis], Oxford University Press, Oxford, (1998).

29. Baig, S. and Rehman, F., [Signal modeling using singular value decomposition. In Advances in Computer, Information, and Systems Sciences, and Engineering], Springer, Netherlands, (2006).

30. Manly, B. F., [Randomization, Bootstrap and Monte Carlo Methods in Biology], Chapman and Hall, London, (1998). 